\title{
Antibacterial Silver Core Spherical Nucleic Acids
}

\author{
Clayton H. Rische, Aakriti Goel, Aleksandar F. Radovic-Moreno, and Sergei M. Gryaznov
}

Exicure, Inc., 8045 Lamon Ave Suite 410, Skokie, IL 60077 USA

\begin{abstract}
Bacterial drug resistance (DR) has the potential to be one of the most significant medical challenges of the $21^{\text {st }}$ century. Infections involving DR bacterial strains such as methicillin-resistant Staphylococcus aureus (MRSA) or DR Acinetobacter baumannii spread in hospital and community settings and are associated with an increased risk of death. New therapeutic strategies and modalities are needed to treat these DR infections, especially for DR Gram-negative infections. Here, we describe silver-core spherical nucleic acids (Ag-SNAs) as a broad-spectrum antibacterial. Ag-SNAs leverage the broad spectrum antibacterial activity of silver nanoparticles (Ag-NPs), while exhibiting significantly lower (30-fold) minimum inhibitory concentration (MIC) values in comparison to Ag-NPs and reduced toxicity (14-fold) to mammalian cells. Ag-SNAs demonstrate low nanomolar MICs against both DR Gram-negative and DR Gram-positive bacteria in vitro and reduced in vitro toxicity toward human cells vis-à-vis non-derivatized silver nanoparticles. In a topical in vivo wound model involving a multi-DR, clinically-isolated, Gram-negative A. baumannii strain, Ag-SNAs significantly reduced bacterial load as compared to controls (1.51-fold $\log _{10}$ reduction). Our findings open up possibilities for the further development of Ag-SNAs as new antibacterial agents, particularly against challenging DR Gram-negative infections.
\end{abstract}

\section{Introduction}

There has been relatively limited development in the field of antibiotics for the past 10 years, with new drugs often accompanied by new bacterial drug resistance (DR) [1]. Many current antibiotics have primarily been sourced from naturally occurring biochemicals, and if past naturally-sourced antibiotics serve as an example, resistance to new drugs appears inevitable, if only delayed [2-4].

Silver nanoparticles (Ag-NPs) [5] and silver ion [6] based treatments have been explored due to their broad spectrum antimicrobial activity, intrinsically low propensity for DR, and ability to synergize with existing antibiotics. However, successful clinical translation of silver-based antibiotics has often been hindered due to challenges with toxicity, formulation, biodistribution, and pharmacokinetics. Prior experiments have been devoted to addressing these challenges through the use of polymer coatings, functionalization with antibiotics, and as embedded constituents of materials such as bandages [7-9]. Due to the small size and relative ease of synthesis, the potential uses and applications of silver nanoparticles extend beyond antimicrobials and into areas such as plasmonic sensors, biological imaging, and microscopy $[10,11]$.

Other approaches to antibiotics have begun to explore the use of antisense RNAs, synthetic oligonucleotides, and other nucleic acid-based molecules [12-14]. By targeting specific genes, essential biological functions in bacteria can be disrupted, leading to cell death or reduction of drug-resistance. These strategies also face difficulties, primarily when tackling delivery of the nucleic acid to the target within bacterial cells. Additionally, there are challenges posed by using compounds such as antisense RNAs, which may present unintentional off-target effects [15]. Despite the success of many of these approaches in vitro, there remains to be seen a successful clinical application of gene modulation in bacteria beyond animal models [16]. Other approaches, such as treatment with peptide nucleic acids, face challenges in uptake and biological half-life, despite resisting enzymatic degradation within cells [17]. An intended target of such therapies has been the acyl carrier protein (acpP), an essential gene in the synthesis of fatty acids for bacterial membranes and polyketides [18].

Spherical nucleic acids (SNAs) are oligonucleotides arranged radially around a core template. SNAs have applications in imaging [19] and gene regulation in eukaryotic cells [20,21], or as DNA probes capable of detecting a wide range of specific biological targets [22]. SNAs have many properties which make them useful in biological settings, including low toxicity, high solubility, protein binding, and intracellular delivery, and have found applications in intracellular mRNA detection and immunomodulation [23]. To date, SNA constructs have been formulated with templates consisting of gold nanoparticles $[19,20]$, small unilamellar vesicles [24] and silver nanoparticles [25]. We hypothesized that the attractive biological properties of SNAs, together with their potential for clinical translation 
including amenability to large-scale manufacturing, could be a way to address some of the hurdles to using silver-based antibacterials.

This work describes the design, synthesis and properties of antibacterial silver-core SNAs (Ag-SNAs, Scheme 1). These structures are a novel formulation of Ag-NPs that leverage the antibacterial properties of silver, but with significantly enhanced potency (up to 30-fold lower MIC) and reduced toxicity (14-fold lower) when compared to Ag-NPs. In addition, Ag-SNAs display broad-spectrum activity against DR Grampositive and DR Gram-negative organisms, including a DR Gram-negative clinical isolate. We have evaluated Ag-SNA interactions with methicillin-resistant Staphylococcus aureus strain 252 (MRSA) and two strains of Acinetobacter baumannii, strain AYE and a multi-DR clinical isolate, termed UNT086-1, as a representative set of DR organisms. These three bacteria represent some of the more persistent Grampositive and negative infections currently observed in the clinic $[26,27]$. Another useful property of silver is its ability to synergize with antibiotics [28]. Consequently, we also evaluated whether Ag-SNAs enhanced the activity of ampicillin, a commonly used systemic antibacterial. Individuals with drugresistant superficial infections $[1,29]$ or those at increased risk of infection (such as burn injury patients or the elderly) [30,31] may benefit from antibiotics with these properties.

\section{Experimental Section}

\subsection{Bacterial Cell Culture}

A. baumannii strain AYE and methicillin-resistant Staphylococcus aureus strain 252 (MRSA-252) were obtained from ATCC. The clinical strain, A. baumannii UNT086-1, was contributed from Pre-Clinical Services at the University of North Texas Health Science Center. Cell cultures were stored via Petri dish culture on trypticase soy agar (TSA (Teknova)) at $4^{\circ} \mathrm{C}$. Mueller Hinton broth (MHB (Becton, Dickinson and Co.)) was used for all in vitro liquid cultures, which were grown at $37^{\circ} \mathrm{C}$ on a Thermo Scientific MaxQ 4000 orbital shaker ( $225 \mathrm{rpm})$ for overnight and intermediate time periods.

\subsection{Oligonucleotide Selection, Synthesis, and Treatment}

Oligonucleotide sequences were originally selected using a BLAST search to analyze matches for antisense sequences targeting the acyl carrier protein (acpP) gene in A. baumannii AYE. This gene was initially selected due to its importance in the synthesis of fatty acids for bacterial membranes. Additional screening was conducted using BLAST to ensure minimal off-target matches in humans. 3'-thiolfunctionalized sequences were then synthesized using solid phase phosphodiester or phosphorothioate chemistry on a MerMade 48 (Bioautomation). Protected DNA bases and related modifications were acquired from ChemGenes. Synthesized oligonucleotide mass and quality was verified via MALDI-TOF and concentrations were verified using UV-visible spectroscopy. Each oligonucleotide was synthesized in the $3^{\prime}$ to $5^{\prime}$ direction, beginning with a $3^{\prime}$ distal thiol group, followed with two successive 18-ODimethoxytritylhexaethyleneglycol,1-[(2-cyanoethyl)-(N,N-diisopropyl)]-phosphoramidite ("spacer-18") molecules which linked to the oligonucleotide sequence. A $1 \mathrm{~mL}$ solution composed of approximately 150 $\mu \mathrm{M}$ of oligonucleotide and $50 \mathrm{mM}$ of DTT was prepared in phosphate buffer solution (Sigma Aldrich). After allowing the solution to incubate at room temperature for 20 minutes, the volume was washed with a 1:2 ratio of ethyl acetate, 99.5\% (VWR). After washing three times, the solution was placed on a shaker table for 2 hours. The oligonucleotide was then purified using NAP desalting columns (GE Healthcare). The unconjugated thiol sample quality was evaluated using a 5,5'-dithiobis-(2-nitrobenzoic acid) (DTNB (Sigma Aldrich)) test. A solution of $30 \mu \mathrm{M}$ of oligonucleotide, $3.2 \mathrm{mM}$ DTNB was created using $0.1 \mathrm{M}$ phosphate buffer, $\mathrm{pH}$ 8. After allowing samples to sit for 15 minutes at room temperature, a DTNB blank was used to establish a baseline on UV-vis, prior to reading the samples between 300-600 $\mathrm{nm}$. Sample concentration was determined using the extinction coefficient of ionized 2-nitro-5thiobenzoate $\left(\varepsilon=13,700 \mathrm{M}^{-1} \mathrm{~cm}^{-1}\right)$. Oligonucleotides were mixed with silver nanoparticles to synthesize silver spherical nucleic acids. Several oligonucleotide sequences were initially synthesized, however due to later difficulties in SNA synthesis, this was later narrowed down to a scramble sequence and two target sequences, one of which presented superior loading on the surface of silver nanoparticles. The phosphorothioate (PS) DNA sequence which presented the best SNA loading was: 5'- 
GCAAATCAAACGCCACAAG-3'. We also used a phosphodiester (PO) version of this sequence and a scramble sequence: 5'-TGACCCTACCCTAGTCCCA-3'.

\subsection{Spherical Nucleic Acid Synthesis}

Preparation of the Ag-SNAs was carried out using prior work with silver [25] and gold $[19,20]$ cores as a basis. For PS-Ag-SNAs, methoxyl poly(ethylene glycol) thiol (PEG-SH, MW = $2 \mathrm{kD}$ (Nanocs)) was added in 500 -fold excess of $10 \mathrm{nM}$ Ag-NPs (US Research Nanomaterials) in 0.1\% Tween-20 and $10 \mathrm{mM}$ phosphate buffer. This was necessary to prevent uncontrolled lateral association of PS oligonucleotides with the particle surface, and ensure that only the $3^{\prime}$-thiol group was the only sulfur atom that could interact with the particle surface. The particles were then incubated at $45^{\circ} \mathrm{C}$ on a shaker for 1.5 hours. After incubation, the thiol-oligonucleotide was added to the particles at 2000-fold excess of the $10 \mathrm{nM}$ particle concentration, and incubated at $45^{\circ} \mathrm{C}$ for 1.5 hours prior to addition of sodium chloride $(500 \mathrm{mM}$ in solution). The particles were incubated overnight at $45^{\circ} \mathrm{C}$. The following day, particles were centrifuged and washed in water 3 times, with the final suspension of particles in $0.0067 \mathrm{M}$ phosphate buffered saline (PBS, Thermo Scientific). PO-Ag-SNAs did not utilize a PEG backfill, as it was not necessary to prevent the oligonucleotide from associating with the particle surface. The synthesis process for $\mathrm{PO}-\mathrm{Ag}$ SNAs was identical to that of PS-Ag-SNAs, however it did not include PEG-SH, was incubated after the addition of oligonucleotide for 3 hours, and stabilized with $250 \mathrm{mM}$ of sodium chloride in solution. The above methods were also used to synthesize PS- and PO-Au-SNAs. Particle concentration was determined using UV-visible spectroscopy.

\subsection{Growth Curves of A. baumannii AYE, UNTO86-1, and MRSA}

Cultures were grown in 96-well plates (Corning) on a rotary shaker $\left(225 \mathrm{rpm}\right.$ ) overnight at $37^{\circ} \mathrm{C}$. Plates were prepared by placing a 1:2 serial dilution of Ag-SNAs across a span of wells ( $50 \mu \mathrm{L}$ in each well), then adding $50 \mu \mathrm{L}$ of bacterial cells at $10^{4}$ cells $/ \mathrm{mL}$. Several wells of media-only, an ampicillin standard curve, and some untreated wells containing only inoculated bacteria were included on each plate. Each plate tested also contained a dilution comparison of Ag-NPs and unconjugated oligonucleotides. After incubation, the optical density (OD900) of bacterial growth was assessed using a plate reader. $900 \mathrm{~nm}$ readings were utilized due to interference on the order of 0.15-0.25 abs from the Ag-SNAs when reading samples at $600 \mathrm{~nm}$. Two groups of Ag-SNAs were tested, one with PS backbone oligonucleotides, and one with PO backbone oligonucleotides. Wells which displayed no visible or detectable growth were verified with colony forming unit (CFU) counts on TSA. Dilutions were created using wells with detectable growth, no growth, untreated wells, and ampicillin-treated wells. The dilutions were $10^{-6}, 10^{-7}, 10^{-8}$, and $10^{-9}$ of the contents of each well sampled in CFU analysis. $5 \mu \mathrm{L}$ of each dilution was plated on the agar and allowed to grow overnight at $37^{\circ} \mathrm{C}$ before counting. This evaluation was designed as a quick assessment and confirmation of apparent MIC concentrations of Ag-SNAs with the bacterial cells. These same methods were also employed to examine PS- and PO-Au-SNAs and multiple PS-Ag-SNAs used to compare different oligonucleotide sequences with $A$. baumannii AYE.

\subsection{Ag-NP and PS-Ag-SNA Kill Curve with A. baumannii UNTO86-1}

A kill curve was determined from observing the growth of $A$. baumannii UNT086-1 challenged with 5 $\mathrm{nM}$ of PS-Ag-SNAs, $5 \mathrm{nM}$ Ag-NPs, and a $26.2 \mu \mathrm{M}$ gentamicin control. Bacteria were added to dilution curves of particles and antibiotic on a 96-well plate, with a final concentration being $10^{7}$ cells $/ \mathrm{mL}$ after being plated. Cells were grown in $\mathrm{MHB}$ at $37^{\circ} \mathrm{C}$. The effective kill curve of the pathogen was determined by obtaining CFU counts over an 8 hour period. Samples were diluted by a factor of $10^{3}$ in $\mathrm{MHB}$ and plated once every hour during the course of the experiment on TSA. After determining the CFU/mL, the silver particle data was plotted and compared to the antibiotic and untreated controls.

\subsection{Membrane Integrity of A. baumannii AYE Treated with PS-Ag-SNAs}


One nanomolar of PS-Ag-SNAs or Ag-NPs was incubated with A. baumannii AYE $\left(10^{5}\right.$ cells $\left./ \mathrm{mL}\right)$ in $10 \mathrm{~mL}$ of TSB for 4 hours. Samples were washed three times with $0.85 \% \mathrm{NaCl}$ solution, with a final solution volume of $10 \mathrm{~mL}$. A LIVE/DEAD Baclight kit (Invitrogen) was used to stain the washed cells using SYTO 9 and propidium iodide. Five microliters of each sample (included untreated control) was taken and placed on glass slides obtained from VRW. Samples were then heat-fixed and imaged with an Olympus IX71 inverted microscope using a Photometrics CoolSnap HQ2 camera.

\subsection{TEM Images of A. baumannii AYE and PS-Ag-SNAs}

Samples were prepared for TEM by inoculating $150 \mathrm{~mL}$ of trypticase soy broth with $10^{4}$ cells $/ \mathrm{mL}$ of $A$. baumannii AYE and adding $1 \mathrm{nM}$ of PS-Ag-SNAs. After overnight incubation, the sample was centrifuged at $7000 \mathrm{rpm}$ for 5 minutes and washed (repeated three times). For the final wash, samples were resuspended with a fixing solution of $1 \%$ glutaraldehyde, $4 \%$ formaldehyde in $0.15 \mathrm{M}$ PIPES buffer, $\mathrm{pH} 7.3$ (Electron Microscopy Sciences). After the final centrifugation cycle, the supernatant was removed and gently replaced with fresh fixing solution, so as not to disturb the pellet. For TEM imaging samples were shipped to Paragon Bioservices (Baltimore, MD), where samples were resuspended and centrifuged in 0.1 $\mathrm{M}$ PIPES. The samples were then resuspended and embedded in $5 \%$ low melt agarose in $0.2 \mathrm{M} \mathrm{PIPES}$, and placed on ice to solidify. Each sample was then washed three times in $0.1 \mathrm{M}$ PIPES (10 mins/wash) and fixed for 1 hour with osmium tetroxide in $0.1 \mathrm{M}$ PIPES. After fixing, samples were washed three times with $\mathrm{dH}_{2} \mathrm{O}$ (10 mins/wash). Solutions of $50 \%, 70 \%$ and $95 \% \mathrm{EtOH}$ were then used to wash the samples (10 mins/wash). Two $100 \%$ EtOH washes followed. Next, samples were washed in 30 minute increments, starting with 2:1 100\% EtOH/Spurr's, then 1:1 and 1:2, followed by $100 \%$ Spurr's for 1 hour, then $100 \%$ Spurr's O/N. Samples were then embedded in fresh Spurr's $\mathrm{O} / \mathrm{N}$ in an oven at $65^{\circ} \mathrm{C}$.

\subsection{Fluorescence Images of Ag-SNAs with A. baumannii AYE}

The same Ag-SNA synthesis outlined above was used to create fluorescently labeled PS-Ag-SNAs for treatment against A. baumannii AYE. The oligonucleotide produced from synthesis was identical to that of the previous experiment; however, the strand included a 5' Cy5 molecule (ex/em $=650 / 670 \mathrm{~nm}$, Glen Research). A serial dilution starting at $50 \mathrm{nM}$ PS-Ag-SNAs was prepared on a 96 -well plate ( $50 \mu \mathrm{L}$ per well) prior to addition of $50 \mu \mathrm{L}$ of bacterial cells at $10^{4}$ cells $/ \mathrm{mL}$. A row of untreated cells was also added for comparison. After overnight incubation, $100 \mu \mathrm{L}$ was taken from the $5 \mathrm{nM}$ PS-Ag-SNA or the untreated wells and placed in a microcentrifuge tube. Samples were washed three times in PBS. Five microliters of washed sample was taken and placed on glass slides obtained from VRW. Samples were then heat-fixed and imaged with an Olympus IX71 inverted microscope using a Photometrics CoolSnap HQ2 camera.

\subsection{Synergy of PS-Ag-SNAs and Ampicillin}

The checkerboard method for synergy [32] was used to evaluate MRSA and A. baumannii UNT086-1 growth. A serial dilution of ampicillin was conducted, starting at $673 \mu \mathrm{M}$ with a final volume of $40 \mu \mathrm{L}$ in each well. A concentrated serial dilution of PS-Ag-SNAs was added in $10 \mu \mathrm{L}$ volumes to each well to bring the total volume up to $50 \mu \mathrm{L}$. Inoculum of $10^{4}$ cells $/ \mathrm{mL}$ of MRSA-252 was added at $50 \mu \mathrm{L}$ volume to bring the total volume in each well to $100 \mu \mathrm{L}$. A strip of wells was reserved both for ampicillin-only and PS-AgSNA-only treatments to compare against the mixed wells and establish fractional inhibitory concentrations (FICs). When two therapeutic agents are used together, FIC is defined as the fractional value of an individual antimicrobial agent's MIC. In opposite corners of the plate, untreated controls were placed for growth comparison. Samples were read using the same settings from prior experiments (OD900) and evaluated based on optical growth density.

\subsection{Toxicity Screen of Ag-SNAs, HEK293, and HFK cells}


Flow cytometry was conducted using a Guava easyCyte 8HT from EMD Millipore. HEK293 cells were incubated with $3.75 \mathrm{nM}$ of PS-Ag-SNAs or Ag-NPs for 4 hours before being treated with a propidium iodide and calcein AM stain. Each group contained at least 1,200 counted cells, and data was analyzed using FlowJo. An alamarBlue (Invitrogen) assay was used to evaluate the toxicity of PS-Ag-SNAs and AgNPs in the presence of human foreskin keratinocytes supplied by Northwestern University. Serial dilutions of the particles were created on a 96-well plate using cell culture media. Silver particle concentrations ranged from $10 \mathrm{nM}$ to $0.3125 \mathrm{nM}$, and were incubated with cells for 24 hours at $37^{\circ} \mathrm{C}$ in a VWR Symphony $\mathrm{CO}_{2}$ cell incubator. Following the treatment cells were washed with PBS and $10 \mu \mathrm{L}$ volume of alamarBlue reagent was added directly to cells in culture medium in 96-well plate. Cells were then incubated for 1 to 4 hours at $37^{\circ} \mathrm{C}$ in the cell culture incubator, protected from light. Absorbance was then recorded at $570 \mathrm{~nm}$ using a plate reader. The cell viability data was expressed in terms of the percentage of cells that survived the treatment. Fluorescence microscopy images were obtained with HEK-293 cells incubated with $3.75 \mathrm{nM}$ of fluorescent PS-Ag-SNAs for 4 hours in an 8-well chamber slide prepared with Geltrex basement matrix. Cells were washed 3 times with $0.85 \% \mathrm{NaCl}$ solution before staining. Calcein AM and propidium iodide were used to track cell membrane integrity. NucBlue was utilized to stain each cell nucleus, and Cy5-functionalized oligonucleotides were used to formulate PS-AgSNAs in order to observe the relative locations of the particles. Cells were imaged with an Olympus IX71 inverted microscope using a Photometrics CoolSnap HQ2 camera.

\subsection{Murine Superficial Skin Infection Model with A. baumannii UNT086-1}

For the in vivo assessment of PS-Ag-SNA activity, three sample groups $(n=5)$ and two control groups (colistin, $\mathrm{n}=5$; PBS control, $\mathrm{n}=5$ ) of $\mathrm{CD}-1$ female mice were induced with partial neutropenia (cyclophosphamide, $150 \mathrm{mg} / \mathrm{kg}$ ) 4 days prior to infection. The infection site was the back dorsal surface of each mouse, which was shaved prior to inoculation. The shaved area on each mouse was abraded with a +50 grit emory board, which resulted in a smooth, red, shiny surface with no bleeding. Five microliters of inoculum was then added to each mouse, with first topical application of PS-Ag-SNAs (5 nM), Ag-NPs (5 $\mathrm{nM})$, unconjugated oligonucleotide $(225 \mathrm{nM})$, or colistin $(2.6 \mu \mathrm{M})$ occurring 4 hours post-infection. Mice were treated twice per day for 3 days. The mice were sacrificed 12 hours after the final application of antibiotics, and a tissue sample approximately $2 \mathrm{~cm}^{2}$ in area was excised. Tissue samples were homogenized in $2 \mathrm{~mL}$ of PBS with a Polytron PT10-35 and placed on ice. CFU counts were executed after 18 hours of incubation at $37^{\circ} \mathrm{C}$ on brain heart infusion agar with charcoal. These experiments were conducted by the University of North Texas Health Science Center.

\subsection{Statistical Analysis}

One-way ANOVA with Tukey's HSD was used to determine the significance of the in vivo data, and a ttest was performed on the toxicity data.

\section{RESULTS AND DISCUSSION}

\subsection{Design and Synthesis of Ag-SNAs}

One of the challenges in using silver-based antimicrobials, and Ag-NPs in particular, is unwanted toxicity toward host cells [33]. Therefore, in order to increase the therapeutic index, pharmacokinetic properties, and stability of the Ag-NPs a common strategy has been to coat or functionalize Ag-NPs with various materials, such as chitosan [34]. While chitosan-coated silver nanoparticles demonstrate reduced toxicity, chitosan, as a positively-charged polymer, is subject to biofouling and low colloidal stability in vivo [35]. Ag-SNAs present an oligonucleotide coating on the nanoparticles, which can be cofunctionalized with other molecules. As discussed earlier in the paper, our sequence was designed with antisense functionality in mind, however as we will detail in the following paragraphs, it became clear 
that the sequence was irrelevant to the function of the Ag-SNAs, and that the oligonucleotide performed a far more structural role.

On our PS-Ag-SNAs we co-functionalized PEG strands on the colloidal silver in order to aid in overall oligonucleotide loading and SNA stability. As an added effect, the PEG strands may increase the biocompatibility of Ag-NPs and reduce protein biofouling in vivo [36]. PS-Ag-SNAs were synthesized through a simple stepwise addition of thiol-PEG followed by a large fold-excess of thiol-oligonucleotides to Ag-NPs. PO-Ag-SNAs underwent a similar process, minus PEG and at lower salt concentrations. Pretreating the oligonucleotides to be loaded onto SNA with dithiothreitol (DTT) allowed us to increase the binding availability of oligonucleotides to Ag-NPs [37]. Subsequently, this method made more efficient use of oligonucleotides used to synthesize Ag-SNAs as compared with prior work [25]. Our synthesis utilizes $1 \mathrm{nM}$ of Ag-NPs to every $2 \mu \mathrm{M}$ of oligonucleotides, whereas the work of Lee et al utilizes a ratio of $1 \mathrm{nM}$ of Ag-NPs for every $4.7 \mu \mathrm{M}$ of oligonucleotides. This is a $57 \%$ reduction in synthesis materials which, even if a high loading of 200 oligonucleotides per particle is assumed for Lee et al, results in $4.5 \mu \mathrm{M}$ of wasted oligonucleotide for every $1 \mathrm{nM}$ of Ag-NP material, whereas our particle synthesis results in about $1.95 \mu \mathrm{M}$ of waste. Additionally, our synthesis resulted in a $90 \%$ or greater yield of particles, with losses accounted for during purification. A 5,5'-dithiobis-(2-nitrobenzoic acid) (DTNB) test was utilized to quantify free-thiol and ensure that excess DTT was removed from the oligonucleotides after desalting. We only utilized DTNB-tested oligonucleotides that were within $5 \%$ of the initial concentration of oligonucleotide, according to the reading on the UV-vis spectrometer. If samples presented a significantly higher reading, it indicated that DTT still remained in excess, and the samples needed to be purified by NAP columns once more. Any trace amounts of undetected DTT were likely removed during particle washing. This process is essential, as any toxic effects from DTT would have been undesirable in our experiments. In addition, our synthesis allowed for formulation of Ag-SNAs with variable oligonucleotide backbone chemistries while potentially improving particle stability (Supplemental Table S1). All oligonucleotide sequences were cross-referenced with a BLAST search to decrease the likelihood of offtarget gene regulatory effects in human cells.

\subsection{Particle Characterization}

To verify the successful functionalization of Ag-NPs, we evaluated particle size and charge potential prior to in vitro and in vivo experimentation. Ag-NPs were not stabilized with capping agents and were suspended in $\mathrm{H}_{2} \mathrm{O}$. TEM analysis was used to determine the mean particle diameter and distribution of Ag-SNAs and Ag-NPs used in these experiments. ImageJ software analyzed particle diameter in the TEM images, and the mean size of the Ag-NPs was $25.8 \pm 4.8 \mathrm{~nm}$. The functionalized PS-Ag-SNAs had $45 \pm 4$ oligonucleotides per particle via OliGreen assay and were measured to have a mean diameter of $19.7 \pm$ $4.1 \mathrm{~nm}$. PO-Ag-SNAs contained $43 \pm 5$ oligonucleotides per particle, and our scramble PS-Ag-SNAs were loaded with $48 \pm 3$ oligonucleotides per particle. Based on past work, we expect that PS-Ag-SNAs are loaded with 200-400 PEG strands per particle [38,39].

In addition, our DLS analysis yielded diameter mean averages of $26.26 \pm 3.49 \mathrm{~nm}$ (PS-Ag-SNAs) and $21.94 \pm 0.54 \mathrm{~nm}$ (Ag-NPs). There are some inconsistencies between the TEM and DLS analyses, however this may be explained by sample variability or as an effect of the rigorous synthesis and purification process for Ag-SNAs. The increased DLS diameter of the PS-Ag-SNAs may be an artifact of the oligonucleotides present on the particle surface. Zeta potential measurements indicated the presence of negatively charged oligonucleotides on Ag-SNAs, with a shift in potential from $-2.04 \mathrm{mV}$ to $-16 \mathrm{mV}$ after particle functionalization. Additional data corresponding to the characterization of the particles are found in Supplemental Fig. S1. The characterization results indicated that our particles were functional, stable SNAs, possessing properties consistent with prior data $[19,21]$.

\subsection{Ag-SNAs Antibacterial Activity In Vitro}

To evaluate the antimicrobial potency of the Ag-SNA variants relative to controls, $50 \mu \mathrm{L}$ cultures of $A$. baumannii AYE, $A$. baumannii UNT086-1, or MRSA at $10^{4}$ cells $/ \mathrm{mL}$ were each challenged with $50 \mu \mathrm{L}$ 
variations of Ag-SNAs, Ag-NPs, or unconjugated oligonucleotides (100 $\mu \mathrm{L}$ total). Nanoparticles formulated with PEG but without oligonucleotides were not stable, which was consistent with prior work indicating similar PEG-Ag-NP instability under various conditions.[40] We hypothesized that stable PEG-Ag-NPs require direct synthesis from $\mathrm{AgNO}_{3}$ and capping with PEG-SH,[41] therefore we did not test PEG-Ag-NPs. Two groups of Ag-SNAs were utilized: particles with PS oligonucleotides and particles with PO oligonucleotides. The comparison of PS and PO allowed us to observe any potential influence of oligonucleotide backbone chemistry on particle efficacy. Fig. 1a-c shows the growth inhibition as a function of concentration of $A$. baumannii AYE, A. baumannii UNT086-1, and MRSA as detected via optical density using a 96-well plate reader at $900 \mathrm{~nm}$. PS- and PO-Ag-SNAs generally showed a 10-16-fold reduction in MIC (0.3125 nM Ag-SNAs versus $5 \mathrm{nM} \mathrm{Ag-NPs)}$ and IC50 (0.41 Ag-SNAs versus $4.3 \mathrm{Ag}-\mathrm{NPs})$, both for the Gram-positive MRSA and the Gram-negative A. baumannii. While all PS-Ag-SNAs used to challenge $A$. baumannii exhibited a 2-fold increase in efficacy over PO-Ag-SNAs, both SNA groups had comparably lower MICs as compared to bare Ag-NPs. Interestingly, PS- and PO-Ag-SNAs displayed similar activity against MRSA. Importantly, neither PS nor PO unconjugated oligonucleotide displayed any activity when used alone against the bacterial cells.

Another important implication was that PS- and PO-Ag-SNAs performed comparably while the PO-AgSNAs possessed no PEG, minimizing any potential impact made by including PEG molecules on silver activity. Additionally, while PEG-Ag-NPs were attempted by our group, we found that they could not be synthesized and purified in stable state, particularly under media or increased salt conditions. These observations indicated to us the decreased need and relevance of a PEG-Ag-NP comparison. We also conducted preliminary experiments varying the PEG length used to stabilize the PS-Ag-SNAs. Shorter PEG chains ( $2 \mathrm{kDa}$ and $5 \mathrm{kDa}$ ) had higher potency than a longer PEG chain (15 kDa) (Supplemental Fig. S9). This is consistent with the view that direct interaction of the PS- and PO-Ag-SNAs with the bacteria is important to their enhanced activity. Additionally, the Ag-SNA variants resist dissolution of silver ions and aggregation more readily than Ag-NPs (Supplemental Table S1), which results in a more efficient and lethal exposure of silver ions to bacterial membranes, according to previous work. [42]

To evaluate the kinetics of antibacterial action, we performed a kill-curve experiment (Fig. 1d). PS-AgSNAs effectively eliminated the bacteria after 5 hours of incubation, with no detectable growth via CFU by the 7-hour mark. In contrast, Ag-NPs did not eliminate the bacteria by the end of the experiment ( 8 hours) and were slower to treat the infection than PS-Ag-SNAs. Overall, a marked gap in efficacy existed between both groups of Ag-SNAs and Ag-NPs, particularly in the case of the DR Gram-negative bacteria. This is a clear indication that the SNA construct is important to improving the functionality of silver nanoparticles as an antimicrobial.

We synthesized batches of gold-core ( $\mathrm{Au}$ ) SNAs utilizing the same methods as we employed for PSand PO-Ag-SNAs. PS- and PO-Au-SNAs both contained between 90-100 oligonucleotides per particle. The Au-SNAs were used to challenge A. baumannii AYE along with an ampicillin positive control (Fig. 1e). The results indicate that without the silver core, the particles do not kill bacteria through any means related to the functionalized oligonucleotides. This, along with the lack of activity brought upon by the free oligonucleotides seen in Fig. 1a-c, indicates that there is no bacterial antisense activity attributable to using these oligonucleotides in SNA format.

Lastly, we confirmed whether different sequences used on the PS-Ag-SNAs would impact efficacy. In Figure 1f, we compared PS-Ag-SNAs synthesized with the sequence discussed previously and with a scrambled control shown not to match any significant targets in A. baumannii AYE. The results further support that hypothesis that the oligonucleotide sequence is irrelevant when considering the impact of antisense interactions.

\subsection{PS-Ag-SNAs Interact with Bacterial Cell Membranes}

We hypothesized that the differences in activity could be the result of differences in how the PS-AgSNAs interacted with bacteria. Accordingly, we sought to understand how the PS-Ag-SNAs interacted with bacteria through microscopy techniques. 
A. baumannii AYE membrane integrity in the presence of PS-Ag-SNAs or Ag-NPs was evaluated using a propidium iodide and SYTO 9 stain via fluorescence microscopy (Fig. 2a). Images indicate that treatment with PS-Ag-SNAs resulted in a greater degree of bacterial membrane leakage as compared to Ag-NPs. It is important to note that both PS-Ag-SNAs and Ag-NPs permeabilized the cell membranes extensively enough to allow propidium iodide to stain the DNA inside the cells. However, only the PS-Ag-SNAs appeared to render cells completely inviable, indicated by the low number of cells effectively retaining the SYTO 9 stain. Conversely, the Ag-NP-treated cells appear to possess a greater amount SYTO 9 dye, and therefore greater membrane integrity.

We also used TEM to determine whether the PS-Ag-SNAs penetrated the cytosol or if the particles were purely membrane-associated (Fig. 2b). The TEM images suggest PS-Ag-SNAs associated with bacterial cell membranes. This would indicate that gene regulation was highly unlikely, as cell penetration is necessary to achieve this effect. Furthermore, fluorescence images indicated increased membrane damage by PS-Ag-SNAs, consistent with our current understanding of silver's antimicrobial properties.[43] The enhanced antimicrobial effects of PS-Ag-SNAs are thus potentially a result of improved particle-bacteria contact.[44] TEM images of Ag-NPs were not included in our study as prior work has already well-described these interactions and serves as a good comparison to our data.[45]

To track the association of PS-Ag-SNAs with bacterial cell populations, fluorescence microscopy was utilized to look at the association of particles and cells. PS-Ag-SNAs functionalized with 5'-Cy5 fluorescent oligonucleotides were mixed with bacteria in vitro. In Fig. 2c, untreated $A$. baumannii AYE cells are shown in comparison with PS-Ag-SNA treated cells. Treated cells demonstrated a strong fluorescence signal that colocalized with the bacteria. Previous works observed similar associations of Ag-NPs and bacteria, indicating a similar association of Ag-NPs and bacterial cells.[46]

\subsection{PS-Ag-SNAs Synergize with Ampicillin}

Silver is a unique antimicrobial in part because of its ability to synergize with commonly used antibiotics [28]. Ag-NPs have been functionalized with ampicillin to attack Gram-negative bacteria by way of metal depletion, which interferes with DNA unwinding [7]. Here, we sought to compare the synergistic effects of PS-Ag-SNAs or Ag-NPs with ampicillin against $A$. baumannii UNT086-1 and MRSA. Our results show that PS-Ag-SNAs are superior to Ag-NPs. Ampicillin alone, to which both bacteria displayed resistance, had MIC values of $10.8 \mathrm{mM}$ and $168.3 \mu \mathrm{M}$ for A. baumannii UNT086-1 and MRSA, respectively. Alone, PS-Ag-SNAs displayed MIC values of $1 \mathrm{nM}$ and $3.125 \mathrm{nM}$ for A. baumannii UNT086-1 and MRSA, respectively. The fractional inhibitory concentration (FIC) is a method to analyze the degree of synergy between two agents. The FIC values of MRSA and A. baumannii UNT086-1 challenged by both groups of silver particles are shown in the graphs of Fig. 3a, b. The MIC values of both PS-Ag-SNAs and ampicillin decrease significantly when used together. Both bacterial groups tested demonstrated a significant 4-fold and 128-fold reduction in effective PS-Ag-SNA and ampicillin MIC values, respectively. We also assessed synergy with vancomycin and ciprofloxacin, showing a 4-fold reduction in MIC value of both antibiotics (Supplemental Fig. S4). The data reveal two things: support of prior work, which demonstrates synergy between antibiotics and silver [47], and demonstration of the enhanced synergistic effects of PS-Ag-SNAs as compared to conventional Ag-NPs in conjunction with antibiotics. This synergy is likely a product of the destabilization of the bacterial membrane by both silver particles and ampicillin, and may include the inhibition of DNA unwinding within damaged cells [42] via the interactions of the silver cores within the SNAs, however more comprehensive evidence is required to confirm this hypothesis.

\subsection{Reduced Toxicity of Ag-SNAs as Compared to Ag-NPs in Human Cell Lines}

Concerns over non-specific host toxicity of silver nanomaterials[33,48] prompted us to probe differences in toxicity more deeply. We evaluated cell membrane integrity with the use of flow cytometry and a propidium iodide stain, which bound to exposed DNA and RNA. Healthy or functional cells were stained with calcein AM. This allowed us to differentiate between silver-damaged cells and unaffected 
cells in vitro. We performed an alamarBlue assay to establish a relative toxicity index for these particles in vitro. The graphs in Fig. 4 outline the difference in toxicity between Ag-SNA variants and Ag-NPs. Flow cytometry (Fig. 4a) measured three distinct HEK-293 cell populations (dead, damaged, or healthy cells) treated with $3.75 \mathrm{nM}$ of PS-Ag-SNAs or Ag-NPs. Ag-NPs killed or damaged approximately $80 \%$ of the total cell population, in contrast to fewer than $40 \%$ for PS-Ag-SNAs (Fig. 4a). This is in contrast with bacterial cell interactions, which demonstrated higher membrane sensitivity to PS-Ag-SNAs.

The viability data (Fig. 4b) show a 14-fold difference between the therapeutic MIC of A. baumannii AYE and the concentration where a statistically significant loss in human foreskin keratinocyte (HFK) viability occurs (estimated at $4.375 \mathrm{nM}$ of PS- and PO-Ag-SNAs). No statistical significance was observed between the two Ag-SNA populations. In comparison, polymyxin antibiotics are known to display some adverse nephrotoxic side-effects if delivered intravenously, with a therapeutic index less than 2.5-fold.[49] Importantly, PS- and PO-Ag-SNAs demonstrate a decrease in mammalian cell toxicity as compared to AgNPs, which is critical in any in vivo applications of the particles. We cannot solely attribute this decrease in toxicity as an effect of PEG functionalization, as the PO-Ag-SNAs do not contain this polymer. This indicates that the mechanisms for decreased toxicity must be primarily associated with another interaction between mammalian cells and Ag-SNAs. The eukaryotic cellular toxicity that is observed is likely due to the same mechanisms as bacterial toxicity: membrane permeabilization and metal depletion.

We theorize that the reduction in toxicity may also be due in part to cellular uptake mechanisms that are associated with SNAs [20,23] (Fig. 5). These images display a HEK-293 cell associated with fluorescently-labeled PS-Ag-SNAs. The SNAs appear to be concentrated in two areas, around the cell and within the cellular membrane, around the nucleus. These preliminary findings may indicate signs of endocytosis, which would be consistent with prior understanding of cellular uptake by SNAs [50], however further investigation is required to determine if this is the mechanism by which the Ag-SNAs exhibit decreased toxicity in vitro. Additionally, has been shown that ionic silver has a less toxic impact when compared to chelated silver [42]. This would be relevant in the case of endosomal uptake, where the Ag-SNAs would be dissolved in an acidic compartment. This process would effectively decrease the ability of the Ag-SNA to interfere with DNA unwinding inside cells.

\subsection{In Vivo Topical Application of PS-Ag-SNAs}

Once we determined in vitro toxicity of the NPs, our next step was to investigate the use of PS-AgSNAs in vivo. The treatment of an A. baumannii UNT086-1 infection in a topical wound was explored. Infections demonstrated a statistically significant response to PS-Ag-SNA treatment with a $\log _{10}$ reduction of 1.51 when compared to the untreated controls (Fig. 6). Comparisons with Ag-NPs $\left(\log _{10}\right.$ reduction = 0.63 ) and unconjugated oligonucleotide $\left(\log _{10}\right.$ reduction $\left.=0.58\right)$ further emphasize the improved efficacy of PS-Ag-SNAs. These results demonstrate that antibacterial activity of these PS-Ag-SNAs extend beyond in vitro examination and is transferable to in vivo examination, specifically for a clinically relevant species of DR Gram-negative bacteria.

\section{Conclusions}

In summary, the introduced PS- and PO-Ag-SNAs represent a new strategy in antibiotic design with applicability against DR Gram-positive and DR Gram-negative organisms. Our data demonstrate that AgNPs functionalized with PS- and PO-oligonucleotides offer a means for Ag-NPs to better interact with the surface of bacterial cells, while improving efficacy and decreasing the toxic effects for the host. The AgSNA also presents the possibility of using low-dose silver synergistically with existing antibiotics, such as ampicillin. Ag-SNAs exhibit a functional, stable, and broad spectrum approach to antimicrobials. Our data suggests that the polyvalent nature of the SNA construct combined with the biocidal nature of silver nanoparticles brings us closer to the possibility of safe and powerful silver-based topical antibiotics. These Ag-SNAs differentiate themselves through their enhanced antimicrobial efficacy, potent antibiotic synergy, and improved control over unwanted toxicity as compared to silver nanoparticles, and may find 
applications in treating clinically challenging, multi-DR infections. We believe our data indicates that the unique properties of the SNA construct are instrumental in bringing about these differences in efficacy and stability.

\section{Animal Care and Use Statement}

The experimental protocols and standards of care for mice involved in the topical infection in vivo study were approved for use under the guidelines and supervision of the University of North Texas Health Science Center's Institutional Animal Care and Use Committee. Experiments were also in accordance with the regulatory guidelines of the U.S. Army Medical Department's Animal Care and Use Review Office. All experiments were conducted within local and federal laws.

\section{Acknowledgements}

We would like to extend thanks to B. Tutterow, A. Schook, V. Hilgenberg, and S. Mix for their excellent technical support. This work made use of the EPIC facility (NUANCE Center-Northwestern University), which has received support from the MRSEC program (NSF DMR-1121262) at the Materials Research Center; the Nanoscale Science and Engineering Center (NSF EEC-0647560) at the International Institute for Nanotechnology; and the State of Illinois, through the International Institute for Nanotechnology. We would also like to thanks W. Weiss and the UNT Health Sciences Center for execution and analysis of the in vivo work, J. Goolsby and Paragon Bioservices for preparing and acquiring the TEM images of bacteria, and we are grateful for the support of the Defense Advanced Research Projects Agency under grant number W911QX-13-C-0070.

\section{References}

[1] C. for D. Control, Antibiotic resistance threats in the United States, 2013, National Center for Health Statistics, U.S. Government Printing Office, Washington, DC, 2013.

[2] Davies, J, \& Davies, D., Origins and Evolution of Antibiotic Resistance, Microbiol. Mol. Biol. Rev. 74 (2010) 417-433. doi:10.1128/MMBR.00016.

[3] H. Ochman, J.G. Lawrence, E. a Groisman, Lateral gene transfer and the nature of bacterial innovation., Nature. 405 (2000) 299-304. doi:10.1038/35012500.

[4] C. Walsh, Molecular mechanisms that confer antibacterial drug resistance., Nature. 406 (2000) 775-781. doi:10.1038/35021219.

[5] R. Bryaskova, D. Pencheva, S. Nikolov, T. Kantardjiev, Synthesis and comparative study on the antimicrobial activity of hybrid materials based on silver nanoparticles (AgNps) stabilized by polyvinylpyrrolidone (PVP), J. Chem. Biol. 4 (2011) 185-191. doi:10.1007/s12154-011-0063-9. 
[6] W.K. Jung, H.C. Koo, K.W. Kim, S. Shin, S.H. Kim, Y.H. Park, Antibacterial activity and mechanism of action of the silver ion in Staphylococcus aureus and Escherichia coli., Appl. Environ. Microbiol. 74 (2008) 2171-8. doi:10.1128/AEM.02001-07.

[7] A.M. Fayaz, K. Balaji, M. Girilal, R. Yadav, P.T. Kalaichelvan, R. Venketesan, Biogenic synthesis of silver nanoparticles and their synergistic effect with antibiotics: a study against gram-positive and gram-negative bacteria, Nanomedicine Nanotechnology, Biol. Med. 6 (2010) 103-109. doi:10.1016/j.nano.2009.04.006.

[8] J. Jain, S. Arora, J.M. Rajwade, P. Omray, S. Khandelwal, K.M. Paknikar, Silver nanoparticles in therapeutics: Development of an antimicrobial gel formulation for topical use, Mol. Pharm. 6 (2009) 1388-1401. doi:10.1021/mp900056g.

[9] P. Jena, S. Mohanty, R. Mallick, B. Jacob, A. Sonawane, Toxicity and antibacterial assessment of chitosancoated silver nanoparticles on human pathogens and macrophage cells, Int. J. Nanomedicine. 7 (2012) 1805-1818. doi:10.2147/IJN.S28077.

[10] K.-S. Lee, M.A. El-Sayed, Gold and silver nanoparticles in sensing and imaging: sensitivity of plasmon response to size, shape, and metal composition., J. Phys. Chem. B. 110 (2006) 19220-5. doi:10.1021/jp062536y.

[11] V. Kravets, Z. Almemar, K. Jiang, K. Culhane, R. Machado, G. Hagen, et al., Imaging of Biological Cells Using Luminescent Silver Nanoparticles., Nanoscale Res. Lett. 11 (2016) 30. doi:10.1186/s11671-016-1243-x.

[12] H. Bai, X. Xue, Z. Hou, Y. Zhou, J. Meng, X. Luo, Antisense antibiotics: a brief review of novel target discovery and delivery., Curr. Drug Discov. Technol. 7 (2010) 76-85. http://www.ncbi.nlm.nih.gov/pubmed/20836761 (accessed August 10, 2016).

[13] J. Meng, G. He, H. Wang, M. Jia, X. Ma, F. Da, et al., Reversion of antibiotic resistance by inhibiting mecA in clinical methicillin-resistant Staphylococci by antisense phosphorothioate oligonucleotide, J. Antibiot. (Tokyo). 68 (2015) 158-164. doi:10.1038/ja.2014.132.

[14] L. Good, S.K. Awasthi, R. Dryselius, O. Larsson, P.E. Nielsen, Bactericidal antisense effects of peptide-PNA conjugates., Nat. Biotechnol. 19 (2001) 360-364. doi:10.1038/86753.

[15] M.K. Thomason, G. Storz, Bacterial antisense RNAs: how many are there, and what are they doing?, Annu. Rev. Genet. 44 (2010) 167-88. doi:10.1146/annurev-genet-102209-163523.

[16] L. Good, J.E.M. Stach, Synthetic RNA silencing in bacteria - antimicrobial discovery and resistance breaking., Front. Microbiol. 2 (2011) 185. doi:10.3389/fmicb.2011.00185.

[17] G. WANG, X.S. XU, Peptide nucleic acid (PNA) binding-mediated gene regulation, Cell Res. 14 (2004) 111-116. doi:10.1038/sj.cr.7290209. 
[18] B.L. Geller, K. Marshall-Batty, F.J. Schnell, M.M. McKnight, P.L. Iversen, D.E. Greenberg, Genesilencing antisense oligomers inhibit acinetobacter growth in vitro and in vivo, J. Infect. Dis. 208 (2013) 1553-1560. doi:10.1093/infdis/jit460.

[19] D.A. Giljohann, D.S. Seferos, W.L. Daniel, M.D. Massich, P.C. Patel, C.A. Mirkin, Gold nanoparticles for biology and medicine, Angew. Chemie - Int. Ed. 49 (2010) 3280-3294. doi:10.1002/anie.200904359.

[20] M.D. Massich, D.A. Giljohann, A.L. Schmucker, P.C. Patel, C. a Mirkin, Cellular response of polyvalent oligonucleotide-gold nanoparticle conjugates., ACS Nano. 4 (2010) 5641-5646. doi:10.1021/nn102228s.

[21] S. Dhar, W.L. Daniel, D.A. Giljohann, C.A. Mirkin, S.J. Lippard, Polyvalent oligonucleotide gold nanoparticle conjugates as delivery vehicles for platinum(IV) warheads, J. Am. Chem. Soc. 131 (2009) 14652-14653. doi:10.1021/ja9071282.

[22] J.-M. Nam, C.S. Thaxton, C.A. Mirkin, Nanoparticle-based bio-bar codes for the ultrasensitive detection of proteins., Science (80-. ). 301 (2003) 1884-6. doi:10.1126/science.1088755.

[23] A.F. Radovic-Moreno, N. Chernyak, C.C. Mader, S. Nallagatla, R.S. Kang, L. Hao, et al., Immunomodulatory spherical nucleic acids., Proc. Natl. Acad. Sci. U. S. A. 112 (2015) 3892-7. doi:10.1073/pnas.1502850112.

[24] K.L. Young, A.W. Scott, L. Hao, S.E. Mirkin, G. Liu, C.A. Mirkin, Hollow Spherical Nucleic Acids for Intracellular Gene Regulation Based upon Biocompatible Silica Shells, 12 (2012) 3867-3871. doi:10.1021/nl3020846.

[25] J.S. Lee, A.K.R. Lytton-Jean, S.J. Hurst, C.A. Mirkin, Silver nanoparticle - Oligonucleotide conjugates based on DNA with triple cyclic disulfide moieties, Nano Lett. 7 (2007) 2112-2115. doi:10.1021/nl071108g.

[26] P.C. Appelbaum, Microbiology of antibiotic resistance in Staphylococcus aureus, Clin.Infect.Dis. 45 Suppl 3 (2007) S165-S170. doi:10.1086/519474.

[27] L.L. Maragakis, T.M. Perl, Antimicrobial Resistance: Acinetobacter baumannii: Epidemiology, Antimicrobial Resistance, and Treatment Options, Clin. Infect. Dis. 46 (2008) 1254-1263. doi:10.1086/529198.

[28] J.R. Morones-Ramirez, J.A. Winkler, C.S. Spina, J.J. Collins, Silver Enhances Antibiotic Activity Against Gram-negative Bacteria, Sci Transl Med. 5 (2013) 1-21. doi:10.1126/scitranslmed.3006276.Silver.

[29] T.S. Murali, S. Kavitha, J. Spoorthi, D. V. Bhat, A.S.B. Prasad, Z. Upton, et al., Characteristics of microbial drug resistance and its correlates in chronic diabetic foot ulcer infections, J. Med. 
Microbiol. 63 (2014) 1377-1385. doi:10.1099/jmm.0.076034-0.

[30] T. Dai, Y.Y. Huang, S.K. Sharma, J.T. Hashmi, D.B. Kurup, M.R. Hamblin, Topical antimicrobials for burn wound infections., Recent Pat. Antiinfect. Drug Discov. 5 (2010) 124-151. doi:10.2174/157489110791233522.

[31] S. Laube, A.M. Farrell, Bacterial skin infections in the elderly: diagnosis and treatment., Drugs Aging. 19 (2002) 331-342.

[32] M.H. Hsieh, C.M. Yu, V.L. Yu, J.W. Chow, Synergy assessed by checkerboard a critical analysis, Diagn. Microbiol. Infect. Dis. 16 (1993) 343-349. doi:10.1016/0732-8893(93)90087-N.

[33] a Haase, J. Tentschert, H. Jungnickel, P. Graf, a Mantion, F. Draude, et al., Toxicity of silver nanoparticles in human macrophages: uptake, intracellular distribution and cellular responses, J. Phys. Conf. Ser. 304 (2011) 012030. doi:10.1088/1742-6596/304/1/012030.

[34] M. Venkatesham, D. Ayodhya, A. Madhusudhan, N. Veera Babu, G. Veerabhadram, A novel green one-step synthesis of silver nanoparticles using chitosan: catalytic activity and antimicrobial studies, Appl. Nanosci. 4 (2012) 113-119. doi:10.1007/s13204-012-0180-y.

[35] A. Rampino, M. Borgogna, P. Blasi, B. Bellich, A. Cesàro, Chitosan nanoparticles: Preparation, size evolution and stability, Int. J. Pharm. 455 (2013) 219-228. doi:10.1016/j.ijpharm.2013.07.034.

[36] E. Caballero-Díaz, C. Pfeiffer, L. Kastl, P. Rivera-Gil, B. Simonet, M. Valcárcel, et al., The toxicity of silver nanoparticles depends on their uptake by cells and thus on their surface chemistry, Part. Part. Syst. Charact. 30 (2013) 1079-1085. doi:10.1002/ppsc.201300215.

[37] W.W. Cleland, Dithiothreitol, a New Protective Reagent for SH Groups, Biochemistry. 3 (1964) 480-482. doi:10.1021/bi00892a002.

[38] H. Hinterwirth, S. Kappel, T. Waitz, T. Prohaska, W. Lindner, M. Lä Mmerhofer, Quantifying Thiol Ligand Density of Self-Assembled Monolayers on Gold Nanoparticles by Inductively Coupled PlasmaÀMass Spectrometry, (1129). doi:10.1021/nn306024a.

[39] K. Rahme, L. Chen, R.G. Hobbs, M.A. Morris, C. O'Driscoll, J.D. Holmes, et al., PEGylated gold nanoparticles: polymer quantification as a function of PEG lengths and nanoparticle dimensions, RSC Adv. 3 (2013) 6085. doi:10.1039/c3ra22739a.

[40] M. Tejamaya, I. Rö, R.C. Merrifield, J.R. Lead, Stability of Citrate, PVP, and PEG Coated Silver Nanoparticles in Ecotoxicology Media, (n.d.). doi:10.1021/es2038596.

[41] A. Shkilnyy, M. Soucé, P. Dubois, F. Warmont, M.-L. Saboungi, I. Chourpa, Poly(ethylene glycol)stabilized silver nanoparticles for bioanalytical applications of SERS spectroscopy., Analyst. 134 
(2009) 1868-1872. doi:10.1039/b905694g.

[42] K.I. Batarseh, Anomaly and correlation of killing in the therapeutic properties of silver (I) chelation with glutamic and tartaric acids, (n.d.). doi:10.1093/jac/dkh349.

[43] C. Lok, C. Ho, R. Chen, Q. He, W.-Y. Yu, H. Sun, et al., Proteomic analysis of the mode of antibacterial action of silver nanoparticles., J. Proteome Res. 5 (2006) 916-924. doi:10.1021/pr0504079.

[44] O. Bondarenko, A. Ivask, A. Käkinen, I. Kurvet, A. Kahru, Particle-Cell Contact Enhances Antibacterial Activity of Silver Nanoparticles, PLoS One. 8 (2013) 1-12. doi:10.1371/journal.pone.0064060.

[45] I. Sondi, B. Salopek-Sondi, Silver nanoparticles as antimicrobial agent: A case study on E. coli as a model for Gram-negative bacteria, J. Colloid Interface Sci. 275 (2004) 177-182. doi:10.1016/j.jcis.2004.02.012.

[46] B. Das, S.K. Dash, D. Mandal, T. Ghosh, S. Chattopadhyay, S. Tripathy, et al., Green synthesized silver nanoparticles destroy multidrug resistant bacteria via reactive oxygen species mediated membrane damage, Arab. J. Chem. (2015). doi:10.1016/j.arabjc.2015.08.008.

[47] P. Li, J. Li, C. Wu, Q. Wu, J. Li, Synergistic antibacterial effects of $\beta$-lactam antibiotic combined with silver nanoparticles, Nanotechnology. 16 (2005) 1912-1917. doi:10.1088/0957-4484/16/9/082.

[48] S. Kim, J.E. Choi, J. Choi, K.H. Chung, K. Park, J. Yi, et al., Oxidative stress-dependent toxicity of silver nanoparticles in human hepatoma cells, Toxicol. Vitr. 23 (2009) 1076-1084. doi:10.1016/j.tiv.2009.06.001.

[49] S.D. Davis, Activity of gentamicin, tobramycin, polymyxin B, and colistimethate in mouse protection tests with Pseudomonas aeruginosa, Antimicrob. Agents Chemother. 8 (1975) 50-53. doi:10.1128/AAC.8.1.50.Updated.

[50] C.H.J. Choi, L. Hao, S.P. Narayan, E. Auyeung, C.A. Mirkin, Mechanism for the endocytosis of spherical nucleic acid nanoparticle conjugates., Proc. Natl. Acad. Sci. U. S. A. 110 (2013) 7625-30. doi:10.1073/pnas.1305804110. 


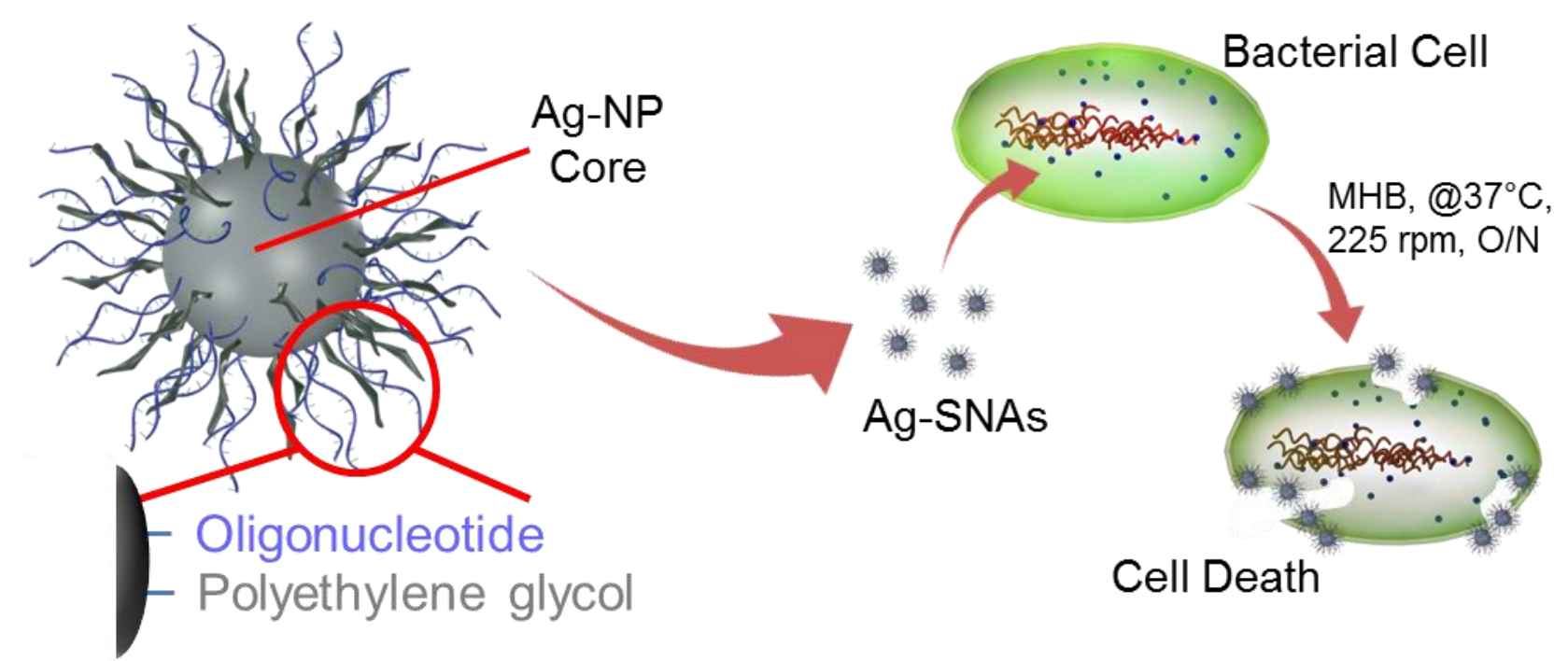

Scheme 1 Silver spherical nucleic acid design and interaction. Using a silver nanoparticle core, a basic PS-Ag-SNA is formulated with the adsorption of $3^{\prime}$-thiololigonucleotide and methoxyl poly(ethylene glycol) thiol. SNAs are known to adhere to or transfect across various types of cell membranes, which serve as the driving force behind a silver-derived SNA. The unique properties of PS- and PO-Ag-SNAs enable the use of these particles as potent antimicrobials. 



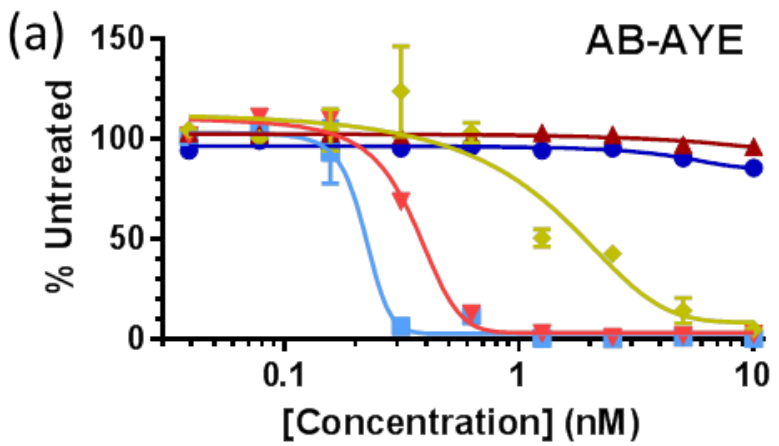

$\rightarrow$ Ag-NPs $\rightarrow$ PS Oligonucleotide

$\simeq$ PS-Ag-SNAS $₫$ PO Oligonucleotide

$\rightarrow$ PO-Ag-SNAS

(b) ${ }^{150} 7$ AB-UNT086-1

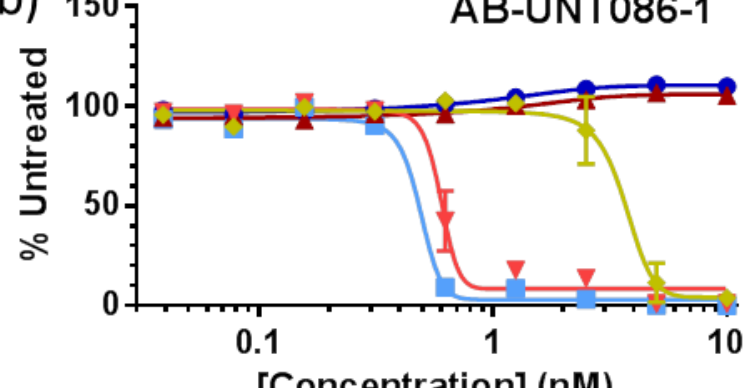

[Concentration] (nM)

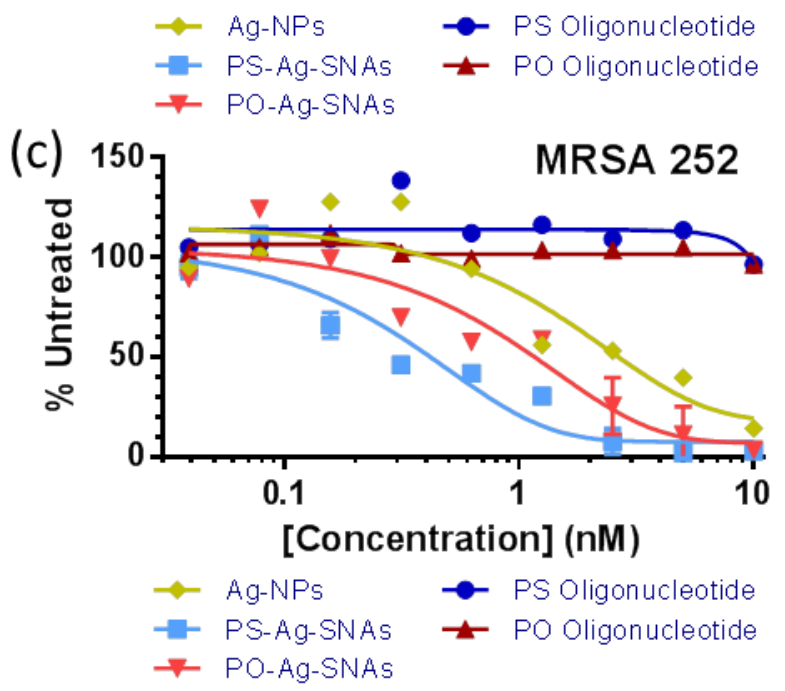

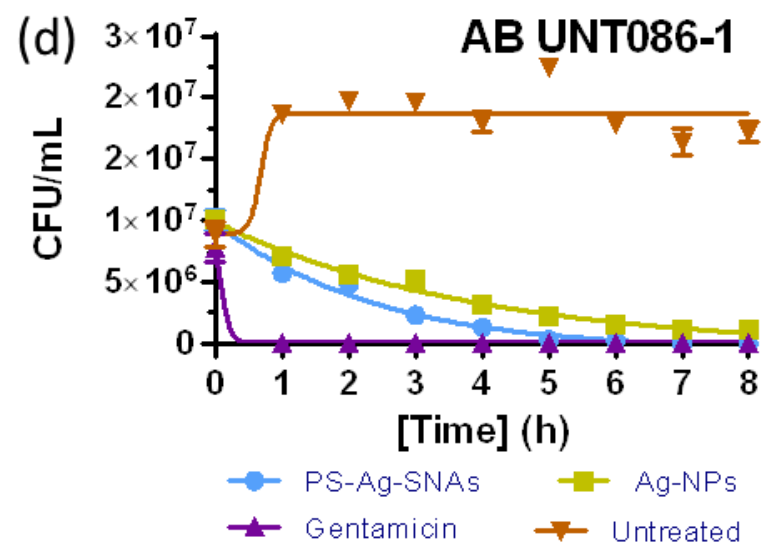

(e)

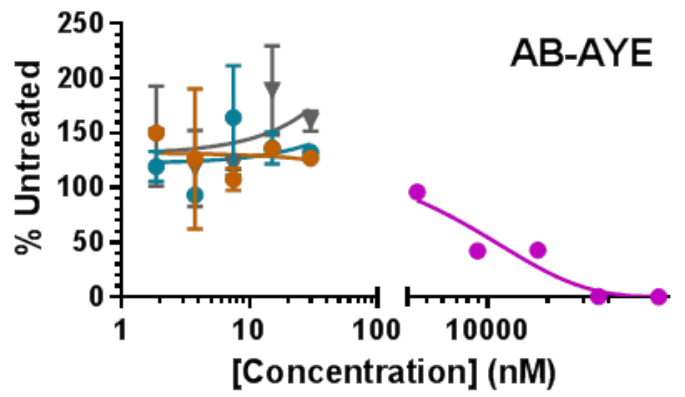

$\rightarrow$ PS-Au-SNAs $\rightarrow$ Scramble Au-SNAs

(f)

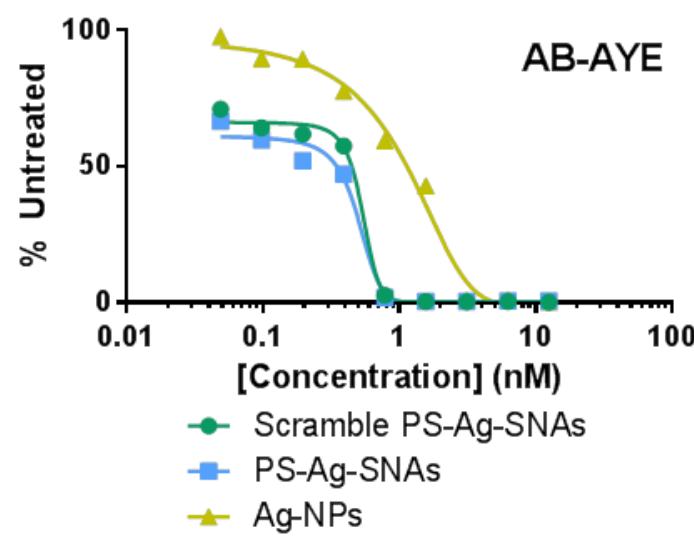

Fig. 1 Growth curves for treated bacteria. (a) A. baumannii AYE treated with PS-Ag-SNAs demonstrates an MIC value of $0.3125 \mathrm{nM}$. The corresponding PO-Ag-SNAs and Ag-NPs displayed MICs of 0.625 and $5 \mathrm{nM}$, respectively. (b) A. baumannii UNT086-1 demonstrated MICs of 0.625, 1.25 , and $5 \mathrm{nM}$ when comparing PS-Ag-SNAs, PO-Ag-SNAs, and Ag-NPs, respectively. (c) MRSA showed a similar reaction to the particles, with MICs of 2.5, 5, and $10 \mathrm{nM}$ for PS-Ag-SNAs, PO-Ag-SNAs, and Ag-NPs, respectively. Unconjugated oligonucleotide did not display activity against any bacteria tested. (d) The time-dependent kill curve of Ag-SNAs and Ag-NPs shows that the SNAs were able to completely eliminate bacteria within 7 hours, whereas Ag-NPs were only able to reduce the bacterial population at the same Ag concentration. (e) Using the same oligonucleotide sequences and a scramble sequence, gold SNAs were formulated and tested against A. baumannii AYE. No growth inhibition was observed, indicating a lack of effective antisense activity on the part of the oligonucleotides. (f) Gene-targeted Ag-SNAs were compared with Ag-SNAs formulated with a randomized scramble control oligonucleotide. There were not any detectable differences stemming between the Ag-SNAs, further suggesting that gene regulation was not a factor in the efficacy of these particles. 
(a) PS- Ag-SNAs

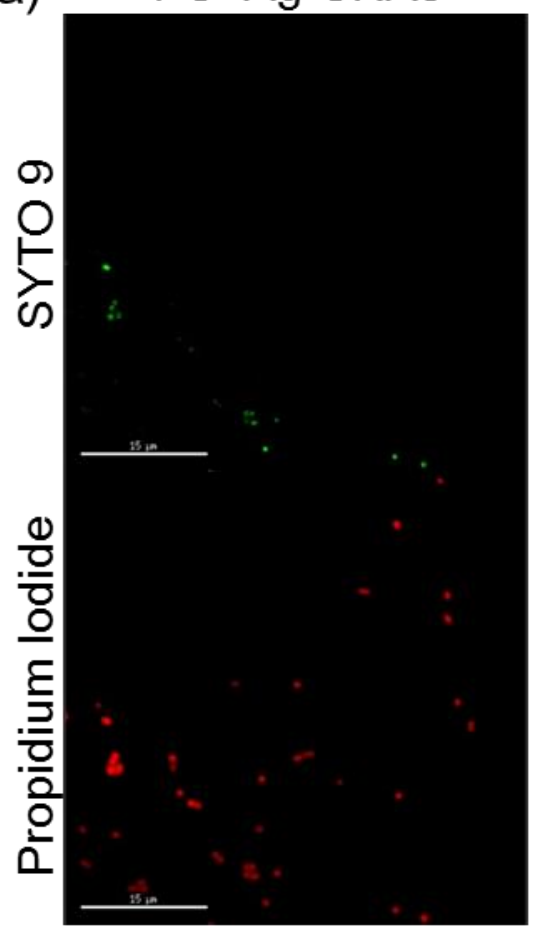

Ag-NPs
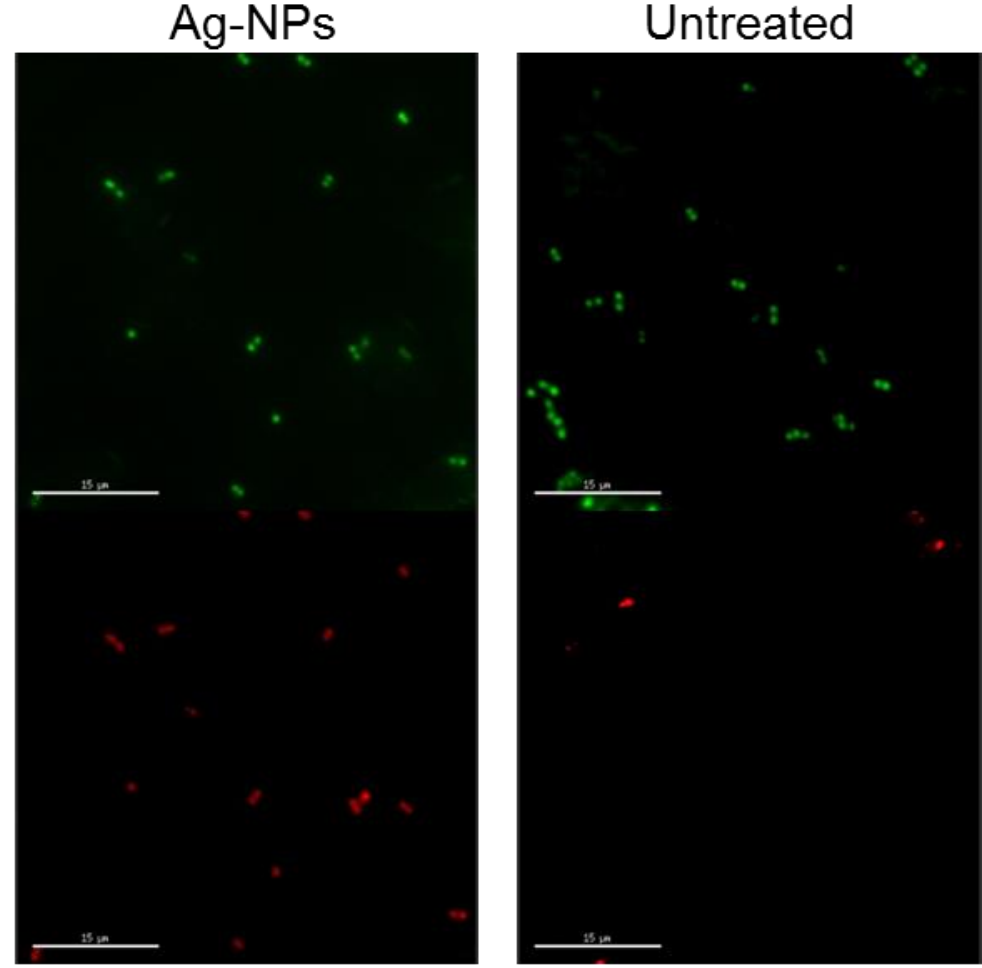

(b)
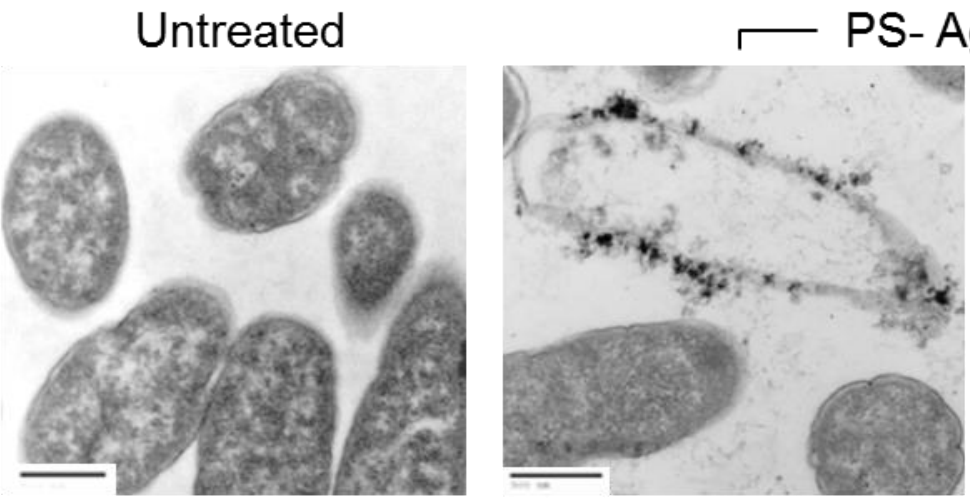

Ag-SNAs $\longrightarrow$

(c)

Untreated

PS-Ag-SNAs
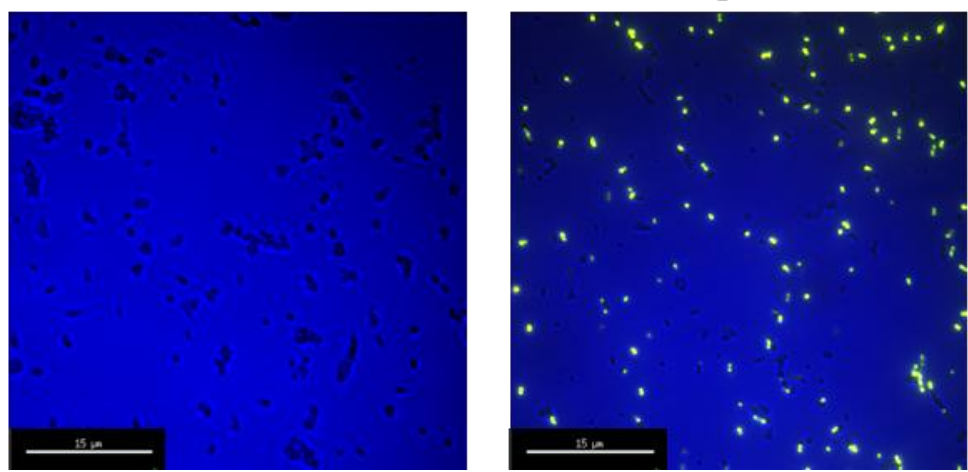

Fig. 2 (a) A. baumannii AYE was stained with propidium iodide and SYTO 9 after treatment with $1 \mathrm{nM}$ of PS-Ag-SNAs or Ag-NPs. Interestingly, PS-Ag-SNAs appeared to damage cell membranes more extensively compared with Ag-NPs. The low number of cells stained with SYTO 9 and large number of cells exclusively stained by propidium iodide indicate significant membrane leakage in the PS-Ag-SNA sample. In contrast, a significant number of cells treated with Ag-NPs retain SYTO 9 stain, and appear to be damaged but viable. (b) TEM images were taken at 30,000x (untreated and left Ag-SNA) and 65,000x (right PS-Ag-SNA) direct magnification. Images display the accumulation of PS-Ag-SNAs on the bacterial membrane. There are signs of membrane deterioration visible in the PS-Ag-SNA affected cell. No significant penetration into the cytosol was observed in the samples. (c) Fluorescence microscopy images of untreated (left) and Cy5-labeled PSAg-SNA treated (right) A. baumannii AYE demonstrate the association of particles with cells in a bacterial population. Interestingly, a universal distribution of particles is not observed. 

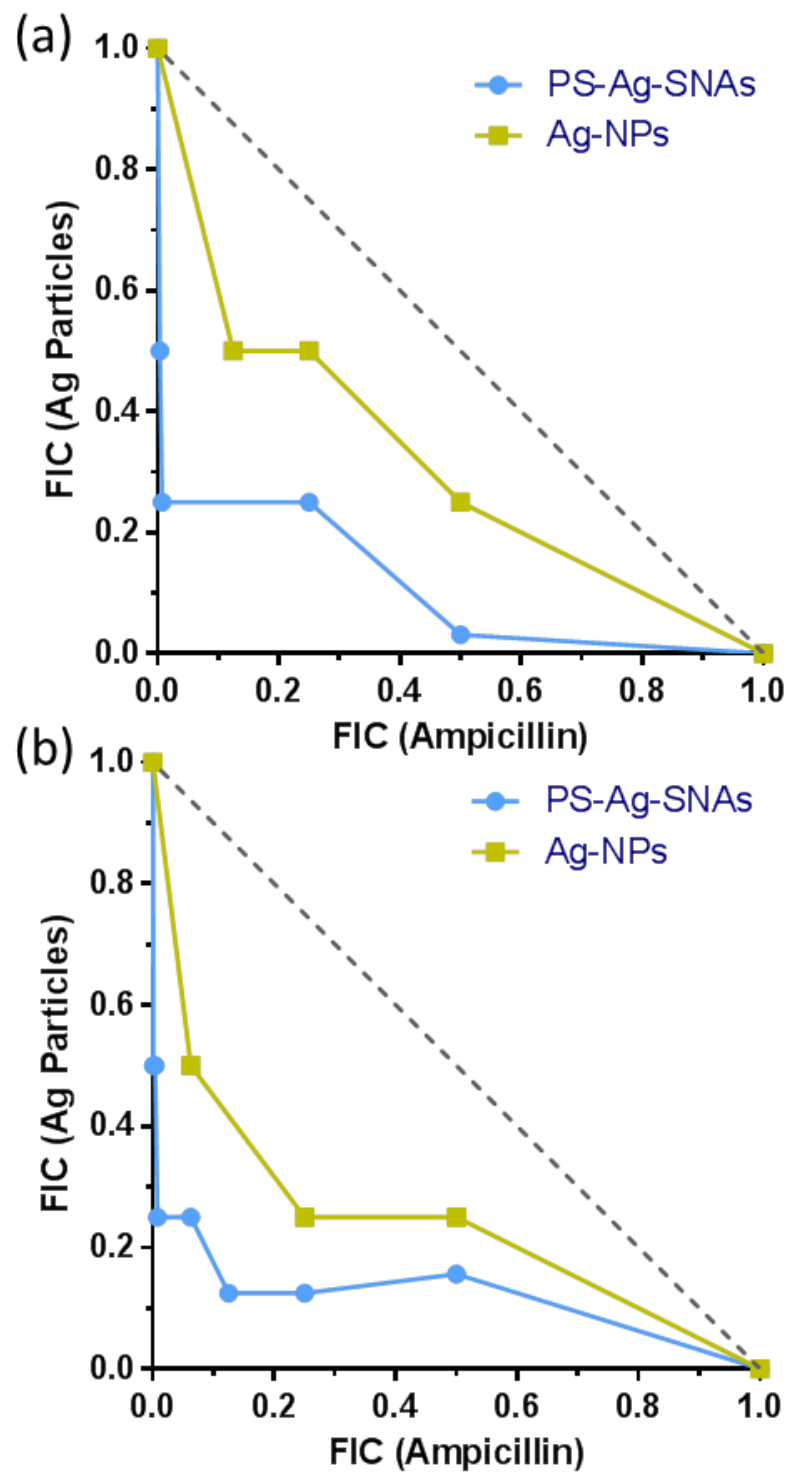

Fig. 3 Ampicillin acts synergistically with PS-Ag-SNAs and Ag-NPs. (a) In MRSA, the best results were seen when PS-Ag-SNAs and ampicillin were dosed at $0.78 \mathrm{nM}$ and $1.32 \mu \mathrm{M}$, respectively. (b) The clinical baumannii strain, UNT086-1, displayed similar results. Only $0.25 \mathrm{nM}$ of PS-Ag-SNAs and $84 \mu \mathrm{M}$ of ampicillin were used at the greatest reduction in MIC with respect to ampicillin. In both tested bacterial strains, these numbers represent 4 -fold and-128 fold decreases in the respective dosage of PS-Ag-SNAs and ampicillin. 


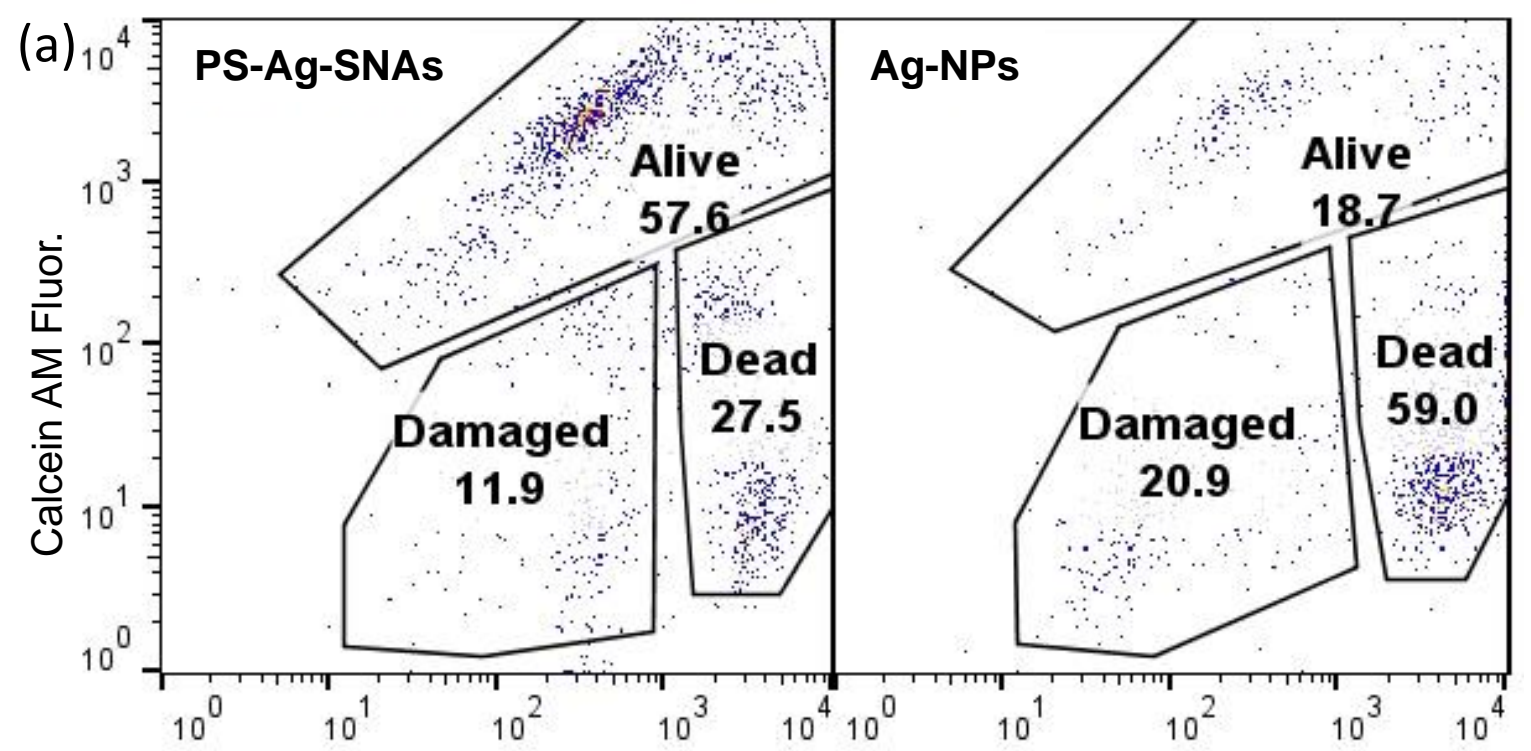

Propidium lodide Fluor.

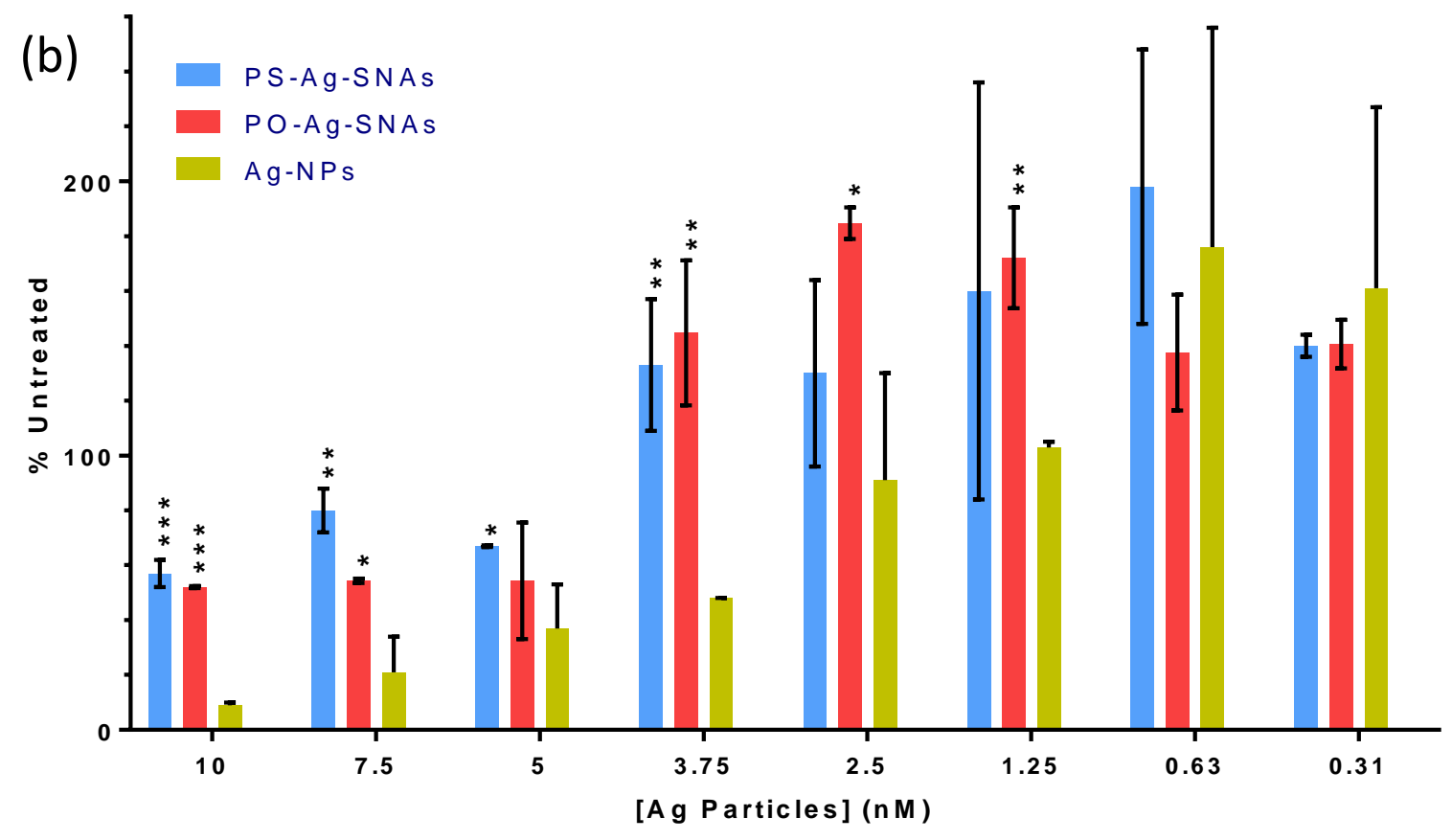

Fig. 4 (a) Flow cytometry was used to investigate cell membrane integrity in the presence of Ag-NPs and Ag-SNAs. HEK-293 cells were treated with 3.75 nM of PS-Ag-SNAs or Ag-NPs. Red stains (propidium iodide) indicate dead cells, whereas green (calcein AM) indicates healthy cells with intact membranes, and a mixture indicates membrane damage on living cells. PS-Ag-SNAs appear to be significantly less damaging to cell membranes. (b) To establish an in vitro toxicity index between the effective treatment of bacteria and toxicity to host cells, human foreskin keratinocytes were treated with various concentrations of Ag-SNA variants and Ag-NPs. A t-test determined that Ag-NPs appeared to have statistically significant $(*=p<0.05, * *=p<0.01, * * *=p<0.001)$ toxic effects on cells at several concentrations compared to PS- and PO-Ag-SNAs. This difference may be attributable to relative SNA stability and more freely available silver ions as compared to the Ag-NPs. 


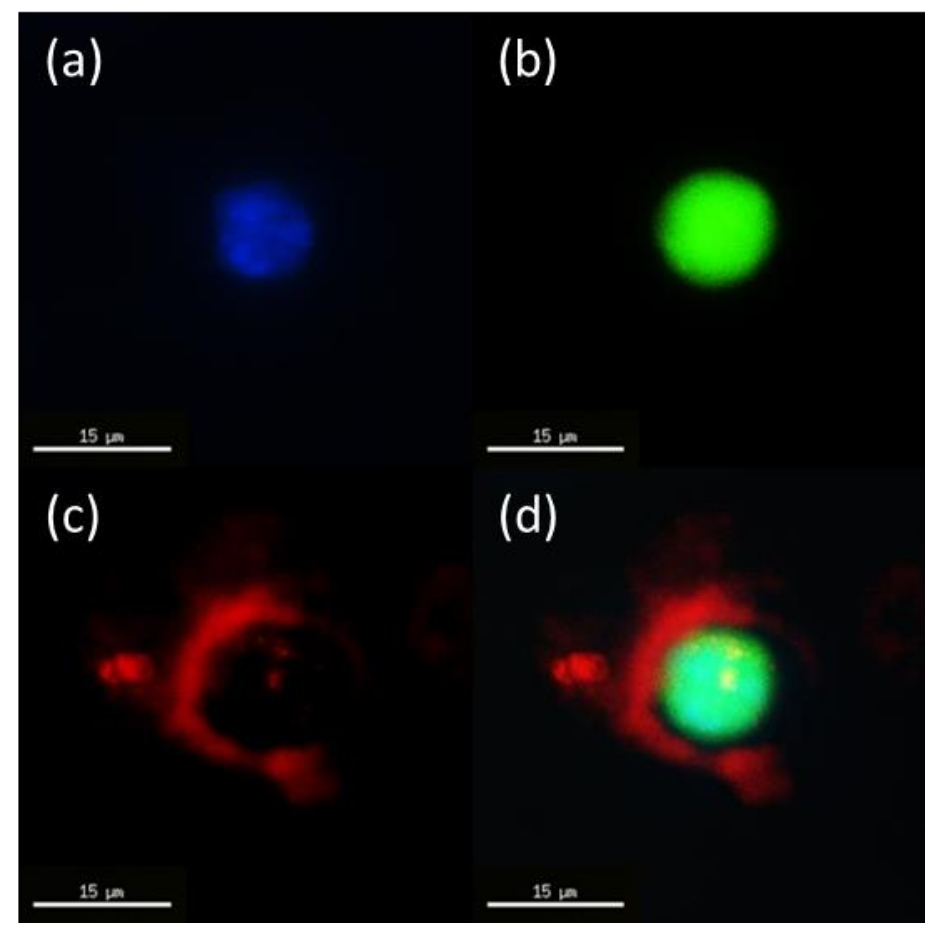

Fig. 5 Flourescent PS-Ag-SNA interactions with HEK-293 cells. The same cell is displayed with a stain of the cell nucleus (a), membrane (b), and PS-Ag-SNAs (c). The merged image (d) displays all stains concurrently. The Cy5 image appears to indicate both membraneassociated particles as well as localized groups of particles around the nucleus. This may be an indicator of endocytosis. 


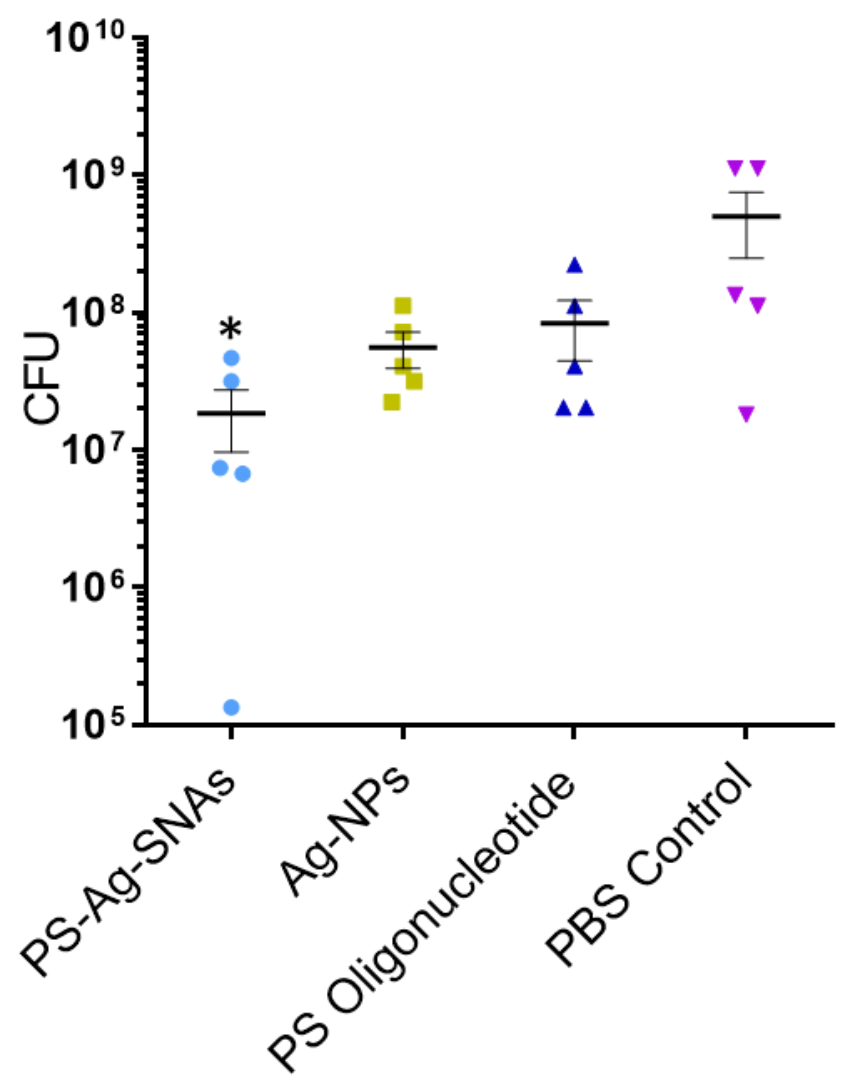

Fig. 6 Topical Infection of $A$. baumannii UNT086-1 treated with Ag-SNAs. Ag-SNAs displayed a decrease in CFUs after treatment, with a significant $\log _{10}$ reduction of $1.51(p<0.05)$ compared to the untreated control. Ag-NPs and unconjugated oligonucleotide did not demonstrate significant activity, with minor $\log _{10}$ reductions of 0.63 and 0.58 . This data appears consistent with our in vitro results, in which Ag-SNAs were the top performer. 